\title{
Processing resources in timing and sequencing tasks
}

\author{
Scott W. Brown and Stephanie M. Merchant \\ University of Southern Maine, Portland, Maine
}

\begin{abstract}
Subjects performed timing and sequencing tasks under separate (single-task) and concurrent (dual-task) conditions in two experiments. The timing task required the subjects to generate a series of 5-sec temporal productions. The sequencing task in Experiment 1 involved verifying reasoning statements that described the ordering of a pair of letters. The task in Experiment 2 involved monitoring a familiar event sequence and detecting omissions in that sequence. Comparisons of single-task and dual-task conditions showed a pattern of bidirectional interference. In each experiment, the concurrent sequencing task caused temporal productions to become more variable and longer. The concurrent timing task interfered with sequencing by lengthening response times to the reasoning statements (Experiment 1) and by lengthening response times to sequence omissions and reducing sensitivity at detecting the omissions (Experiment 2). The results suggest that time perception and sequence perception are related cognitive processes that rely on a common set of attentional resources.
\end{abstract}

The focus of this article is on attentional resources that support timing functions. It is well established that attention is a critically important factor underlying our temporal experience. Attention to time alters the rate at which the subjective flow of time occurs; it influences our ability to gauge the length of intervals, and it affects our memory for the duration of events. The relation between time perception and attention has been the subject of an ever-increasing amount of research over the past 25 years, as investigators have adopted various methodologies to explore the effects of divided attention, resource allocation, expectancy, and automaticity on temporal judgments (e.g., Brown, 1998; Brown \& Bennett, 2002; Fortin \& Masse, 2000; Grondin \& Macar, 1992; Macar, 2002; Macar, Grondin, \& Casini, 1994; Zakay, 1998). This research has led to the development of various theoretical models in which attention plays a prominent role in timing processes (e.g., Block \& Zakay, 1996; Thomas \& Brown, 1974; Zakay \& Block, 1996).

Much of the research on time and attention makes use of the dual-task paradigm. With this method, subjects perform a time judgment task concurrently with a nontemporal distractor task. The distractor task is typically a demanding perceptual or cognitive task, such as pursuit tracking, mental arithmetic, or item recall. The basic finding from this research is a phenomenon called the interference effect (see Brown, 1997, for a review). The interference effect refers to a disruption in timing performance. In comparison with single-task control conditions in which the only task is to judge time, time judgments under dual-task conditions typically show more error and variability. The error in time judgments is typically in the form of a shortening of perceived time, in which less time appears to have passed by, relative to a control condition. It is important to realize that this effect is manifested in different ways, depending on the time judgment method. For verbal estimations or reproductions, time judgments become shorter. However, an opposite pattern occurs with temporal productions, in which subjects attempt to generate a specified interval. Because perceived time is shortened, subjects allow more time to pass before judging that the requisite interval has elapsed. Hence, either (1) shorter verbal estimations or reproductions or (2) longer temporal productions correspond to a shortening of perceived time (see Bindra \& Waksberg, 1956; Brown, 1997; Doob, 1971, pp. 39-44; Fraisse, 1978, pp. 215-217; Zakay, 1993). Subjects' time judgments under distractor conditions may also show increased variability and absolute error. This unreliability may be due, in part, to such factors as shifts in attentiveness between the temporal and the nontemporal tasks, attempts to compensate and adjust for missing temporal information, increased reliance on guessing, and so forth (Brown, 1997, 1998, 2006).

Many theorists explain the interference effect in terms of attentional allocation, in which the concurrent tasks compete for limited processing resources (e.g., Brown, 1997; Casini \& Macar, 1997; Thomas \& Brown, 1974; Zakay, 1989; Zakay \& Block, 1996, 1997). The basic idea is that a person who tries to keep track of time and, simultaneously, perform a distractor task must share resources between the two tasks, with the result that fewer resources than normal are devoted to timekeeping. This reduction in resources interferes with the accumulation of temporal cues, with the consequence that fewer cues than normal are processed

S.W. Brown, swbrown@usm.maine.edu 
and stored in a temporal record of the interval. Time judgments, based on the incomplete record, thus reflect an overall shortening of perceived time. In general, the more resource demanding the distractor task, the greater the degree of interference in timing (e.g., Brown, 1998; Brown \& Boltz, 2002; Fortin, 1999; Fortin, Duchet, \& Rousseau, 1996; Sawyer, Meyers, \& Huser, 1994; Zakay, Nitzan, \& Glicksohn, 1983). However, even relatively light processing demands can substantially disrupt time judgments, which suggests that time perception is very sensitive to the influence of cognitive workload (Brown, 1997).

Researchers have used the concurrent temporal/ nontemporal dual-task paradigm for different purposes. For many researchers, the nature of the timing system has been the primary concern. In this case, all the interest has been centered on how timing performance is influenced by the distractor task. For other investigators, however, the main focus is on performance of the nontemporal task. Much of this work has involved human factors studies of piloting performance in flight simulators (e.g., Bortolussi, Kantowitz, \& Hart, 1986; Casali \& Wierwille, 1983, 1984; Wierwille \& Connor, 1983; Wierwille, Rahimi, \& Casali, 1985; Zakay \& Shub, 1998). These studies have employed the secondary task technique, in which the primary task (flight performance) is maintained at a high level, while any spare capacity is directed to a concurrent secondary timing task (typically, temporal production). Timing performance is taken as an index of the workload demands of the piloting task. Timing is considered to be an ideal secondary task for this purpose, since it is sensitive to primary task demands but usually does not intrude on primary task performance (see Eggemeier \& Wilson, 1991, and O'Donnell \& Eggemeier, 1986, for reviews). In a similar vein, other researchers have advocated using concurrent timing performance as an indicator of the processing demands of various cognitive tasks, including lexical decision (Chastain \& Ferraro, 1997), target monitoring (Shinohara, Miura, \& Usui, 2002), psychometric intelligence (Fink \& Neubauer, 2001), and memory search (Fortin \& Rousseau, 1987).

These two approaches are combined in the present research. We believe that an evaluation of both temporal and nontemporal performance can lead to a better understanding of timing processes. This approach stems directly from resource theory. An attentional resource model implies that if common resources are shared between two tasks, each task receives a reduced amount of attention. Assuming that the two tasks have equal priority and that their resource demands are roughly comparable, one might expect to find a bidirectional pattern of dual-task interference. That is, if a distractor task interferes with timing because of a suboptimal supply of resources, timing should also interfere with the distractor task, for the same reason. The question of bidirectional interference has important implications for understanding the cognitive psychology of time. Resource theorists argue that different tasks may require different types of attentional resources in different compositions and that the degree of interference between concurrent tasks may indicate the extent to which they depend on the same sets of specialized resources (Navon \& Gopher, 1979, 1980; Wickens, 1984). For example, suppose that a distractor task is found that produces bidirectional interference with timing. This outcome suggests that at least some of the resources that are important for the distractor task are also critical for the timing task as well. Mutual interference implies that the two tasks rely heavily on common resources or mechanisms (Navon \& Gopher, 1979). In contrast, suppose that the distractor task produces interference in just one direction. That is, the distractor task interferes with timing, but timing does not interfere with the distractor task. This pattern implies that the two tasks are less related. Resource theory usually attributes asymmetrical interference to a situation involving shared resources that are differentially important for the two tasks (Navon \& Gopher, 1980; Tsang, Shaner, \& Vidulich, 1995; Wickens, 1980). One review revealed that whereas most tracking manipulations affect concurrent shadowing or mental arithmetic tasks, most manipulations of shadowing or arithmetic difficulty fail to affect tracking performance (Wickens, 1980). This asymmetry in interference occurs because the common resources involved may make a larger contribution to one task than to the other, thus making that task more vulnerable to resource competition. All these considerations indicate that interference patterns between concurrent timing and distractor tasks may provide insight as to the nature of the attentional resources involved in time perception.

What do the data show on this issue? Unfortunately, data concerning bidirectional interference in timing are limited. Most experimenters are concerned with timing performance and regard the distractor task only as a means of diverting attention away from time. Consequently, most dual-task timing studies do not even report distractor task performance, nor do they include a single-task control condition involving the distractor task alone. These limitations preclude an evaluation of interference patterns in most studies. However, in a recent review (Brown, 2006), 33 dual-task timing experiments were identified that are not subject to these problems. These experiments measured distractor task performance under both single-task and dual-task conditions and, so, offer some insight into the issue of bidirectional interference. About half of the studies show evidence of bidirectional interference, whereas the other half show interference with the timing task only. The main feature that the bidirectional interference studies have in common is that they tend to involve distractor tasks related to executive cognitive functions. Executive functions (also called frontal lobe functions) are those cognitive processes that regulate behavior. These processes include judgment, planning, coordinating actions, monitoring outcomes, and inhibiting distractions (Fournier, Larigauderie, \& Gaonac'h, 2004; Logan, 1985; Miyake et al., 2000; Royall \& Mahurin, 1996). Thus, dual-task timing experiments that involve attentional tasks emphasizing comprehension (Hiscock, Cheesman, Inch, Chipuer, \& Graff, 1989), arithmetical calculation (Kantowitz \& Knight, 1974), monitoring response output (Brown, 2006), and maintaining information in working 
memory (Franssen \& Vandierendonck, 2002) show evidence of bidirectional interference. This pattern suggests that timing is closely related to executive functioning. As such, it should rely on processing resources that are the same as or similar to those used by other executive tasks.

In the present research, we examined interference patterns between concurrent timing and sequencing tasks. We selected sequencing tasks for two reasons. First, sequencing may be regarded as a basic executive process (Royall \& Mahurin, 1996). Sequencing is an essential feature of the executive tasks of integrating information, coordinating actions, and executing a multistep plan. Given the association between timing and other executive tasks noted above, it seems reasonable to expect that sequencing performance will also be affected by a concurrent timing task. The second reason is that timing and sequencing are conceptually related. Sequencing, by its very nature, is a temporal task. Sequencing involves ordering a succession of objects or events in a series, a process that is fundamentally temporal. Likewise, timing is, in essence, a sequencing task. Various writers have characterized timing as perceiving a series of changing events (Block, 1982, 1990; Brown, 1995; Fraisse, 1963; Gibson, 1975) or segmenting experience into a string of smaller units (Poynter, 1989; Poynter \& Homa, 1983). Basic strategies for marking time include chronometric counting (Mississippi-one, Mississippi-two, etc.), visualizing the movement of a clock's second hand, and tapping a rhythmical pattern (e.g., Brown, Newcomb, \& Kahrl, 1995; Doob, 1971, pp. 164-165; Goldstone, Boardman, \& Lhamon, 1958; Guay \& Wilberg, 1983), all of which involve the generation of event sequences. Thus, both timing and sequencing involve temporal information processing and, so, probably invoke similar cognitive processes or mechanisms. Therefore, we predict a pattern of bidirectional interference - that sequencing interferes with timing (the standard interference effect) and, more critically, that timing also interferes with sequencing. The prediction was tested in two dual-task timing experiments, each involving a different sequencing task. Experiment 1 involved a verbal-reasoning task in which the subjects verified the sequencing of letter pairs. In Experiment 2, the subjects performed a monitoring task to detect errors in a familiar event sequence.

\section{EXPERIMENT 1 Timing and Sequence Reasoning}

The sequencing task selected for the first experiment was based on a reasoning task devised by Baddeley (1968; Baddeley \& Hitch, 1974). The task required the subjects to verify statements that described the ordering of pairs of letters. For example, typical statements would assert $A$ follows $B, B$ precedes $A, A$ does not follow $B$, and so forth. The letters AB (or BA) then followed the statement, and the subjects judged, as rapidly as possible, whether the statement was true or false. The essential feature of the task was that the subjects had to process the temporal verb in the statement (follows, precedes, does not follow, etc.) and then apply that relation to the letter pair. Thus, the task may be construed as being primarily a sequence-reasoning or judgment task.

Method
Subjects
Fifty-nine students ( 5 male, 54 female) enrolled in general psy-
chology classes at the University of Southern Maine participated as
subjects in exchange for extra course credit. The mean age of the
students was 24.1 years ( $S D=8.5$ years).

\section{Apparatus and Stimuli}

An Apple II-GS computer equipped with a Timemaster II H. O. clock card (Applied Engineering) set at an interrupt rate of $1024 \mathrm{~Hz}$ was programmed in Applesoft Basic to present stimuli and record responses. The stimuli for the sequence-reasoning task consisted of a series of items presented one at a time at the center of the computer screen. Each item consisted of a statement concerning the ordering of the letters $\mathrm{A}$ and $\mathrm{B}$, followed by the pair of letters (e.g., the items would appear in the form $A$ follows $B-A B$ ). Half of the statements were true, and half were false. The statements were constructed in such a way as to produce a positive item condition and a negative item condition. In the positive condition, the statements were worded in the affirmative; in the negative condition, they were worded in the negative with the word not (see Table 1). Previous research (Baddeley \& Hitch, 1974) has indicated that this factor produces substantial differences in reasoning speed and, thus, may represent different degrees of task difficulty.

Within the positive and negative conditions, the statements varied in terms of verb type (precedes vs. follows) and active or passive voice (e.g., follows vs. is followed by). These manipulations served to create a wider variation in the structure of the statements and, thus, minimized the possibility that the subjects would become accustomed to judging the same limited set of items over and over. The items also varied in terms of the order in which letters appeared in the statements (in half of the statements, A was mentioned first, and in half of the statements, B was mentioned first). Finally, we also varied the ordering of the letter pair that followed each statement, so that half the statements were followed by an $\mathrm{AB}$ pair and half were followed by a BA pair. All these variations yielded 32 unique combinations that constituted an item pool. The program selected these items randomly until all the items in the pool had been used, at which point the program began the selection process anew. This same randomization procedure was repeated continuously throughout the experiment.

\section{Design and Procedure}

The experimental design was patterned after that used in previous investigations of bidirectional interference in timing (Brown, 1997, 2006). The subjects were tested individually in two sessions separated by $1-2$ weeks. Watches were removed at the beginning of each session. Session 1 consisted of single-task conditions, in which the subjects performed a timing task, the positive version of the sequencing task, and the negative version of the sequencing task. Each task was performed separately. Session 2 was devoted to dual-task conditions, in which the subjects performed the timing task concurrently with each of the two sequencing tasks. In all cases, the tasks were performed for three 2-min trials. The order of the tasks within each session was counterbalanced across subjects.

Table 1

Types of Reasoning Statements Used in Experiment 1

\begin{tabular}{ll}
\hline \multicolumn{1}{c}{ Positive } & \multicolumn{1}{c}{ Negative } \\
\hline A follows B & A does not follow B \\
A precedes B & A does not precede B \\
A is followed by B & A is not followed by B \\
A is preceded by B & A is not preceded by B \\
\hline
\end{tabular}


Note that the single tasks were always performed in the first session and the dual tasks in the second session. This procedure was adopted because of the following considerations: (1) The ordering ensured that the subjects had some basic familiarity with the individual tasks before attempting to perform them under dual-task conditions; (2) the 1- to 2-week interval separating the sessions was probably sufficient to minimize any potential carryover effects; and (3) the subjects did not know what would be required of them in Session 2 , and so practice on the tasks during the intersession interval was unlikely. In any event, any benefit derived by exposure to the single tasks in Session 1 would serve only to make it more difficult to obtain evidence of dual-task interference in Session 2. This procedure may, therefore, underestimate the strength of any bidirectional interference effects that we report.

Each trial was initiated by having the subjects press the Delete key on the keyboard; the end of the trial was signaled by a beeping sound emitted by the computer. The timing task involved serial temporal production. The subjects were instructed to press the space bar on the computer keyboard with their left hand every $5 \mathrm{sec}$ throughout the trial. This procedure has been used in numerous experiments and provides a continuous online measure of timing. The subjects were encouraged to be as accurate as possible in making their timing responses. The sequencing tasks required the subjects to respond to each item with their right hand by pressing the "/" and "** keys located in the extreme upper right part of the keyboard. The subjects lightly rested their fingers on the two keys, which were labeled $T$ (true) and $F$ (false). They were asked to make their responses as quickly and accurately as possible. The next reasoning item appeared on the screen immediately after each response had been made. Thus, the sequencing task was continuously ongoing. The dual-task conditions required the subjects to perform the timing and sequencing tasks simultaneously. The instructions stressed that both of the tasks were equally important and that the subjects were to divide their attention evenly between the two.

\section{Results and Discussion}

Interference would be indicated by a performance decrement from the single-task to the dual-task conditions. For the timing task, the decrement would be in the form of greater variability, lengthening, or error in temporal productions. These properties are represented by coefficient of variation (CV), interresponse interval (IRI), and absolute error scores, respectively. For the sequencing task, a performance decrement would be reflected in longer response times (RTs) and/or less accurate responses. A decrement for both timing and sequencing would constitute evidence of bidirectional interference.

\section{Timing Performance}

The timing data were transformed into three different scores for analysis. The scores were the CV, the mean IRI, and the mean absolute error. These scores provide a comprehensive picture of timing performance under the vari- ous experimental conditions. Responses from the three trials were combined to form the basic unit of analysis. We also conducted analyses including trials as a factor, and the results did not differ substantially from those reported below. The mean scores for the dependent measures are shown in Table 2.

Coefficient of variation. The $\mathrm{CV}$ was formed by dividing the standard deviation of the timing responses by the mean length of the responses. These scores represent a relative measure of timing variability and have been shown to be very sensitive at detecting the influence of distractor task demands (e.g., Brown, 1997, 1998, 2006; Hiscock et al., 1989; Kee, Morris, Bathurst, \& Hellige, 1986). CV scores were calculated for each subject in each condition and submitted to a one-way repeated measures ANOVA comparing the three task conditions (timing only, timing + positive items, and timing + negative items). The task effect was significant $[F(2,116)=22.79, p<$ $\left..001, \eta_{\mathrm{p}}^{2}=.28\right]$. Orthogonal contrasts were used to test for differences between means. Contrast 1 confirmed that variability in timing responses increased from the single-task condition $(M=0.15 \mathrm{sec})$ to the combined dual-task conditions $(M=0.30 \mathrm{sec})[F(1,58)=59.50$, $\left.p<.001, \eta_{\mathrm{p}}^{2}=.51\right]$. This result demonstrates the classic interference effect in timing and fits in with the idea that a distractor task diverts attentional resources away from timing. Contrast 2, comparing the two dual-task conditions, was not significant $(F<1)$. As is shown in Table 2, the positive and negative conditions produced essentially the same degree of timing variability.

Interresponse interval. The mean temporal production (i.e., the average interval between space bar presses) is represented by the mean IRI. The IRI is a directional measure of error, indicating whether there is any consistent trend toward overestimation or underestimation. Many studies have shown that concurrent distractor tasks interfere with timing by shortening perceived duration (see Brown, 1997). As was discussed previously, a shortening of perceived duration is manifested as a lengthening of temporal productions. Thus, one would expect that the concurrent sequence-reasoning tasks would lengthen IRI scores.

Mean IRI scores were computed for each subject and submitted to a one-way repeated measures ANOVA. The task effect was significant $[F(2,116)=9.47, p<$ $\left..001, \eta_{\mathrm{p}}^{2}=.14\right]$. Contrast 1 , comparing the timing-only condition $(M=4.4 \mathrm{sec})$ with the combined dual-task conditions $(M=5.1 \mathrm{sec})$, was significant $[F(1,58)=14.67$, $\left.p<.001, \eta_{\mathrm{p}}^{2}=.20\right]$. This finding that longer temporal productions were associated with the dual tasks supports

Table 2

Mean Scores (and Standard Errors) for Three Timing Performance Measures in Different Task Conditions in Experiment 1

\begin{tabular}{|c|c|c|c|c|c|c|}
\hline \multirow[b]{2}{*}{ Task Condition } & \multicolumn{2}{|c|}{$\begin{array}{l}\text { Coefficient of } \\
\text { Variation }(\mathrm{sec})\end{array}$} & \multicolumn{2}{|c|}{$\begin{array}{l}\text { Interresponse } \\
\text { Interval (sec) }\end{array}$} & \multicolumn{2}{|c|}{$\begin{array}{l}\text { Percentage of } \\
\text { Absolute Error }\end{array}$} \\
\hline & $M$ & $S E$ & $M$ & $\overline{S E}$ & $M$ & $S E$ \\
\hline Timing only & 0.15 & 0.01 & 4.4 & 0.2 & 28.0 & 2.2 \\
\hline Timing + positive reasoning & 0.31 & 0.02 & 5.1 & 0.2 & 36.8 & 2.4 \\
\hline Timing + negative reasoning & 0.29 & 0.02 & 5.1 & 0.2 & 36.2 & 2.8 \\
\hline
\end{tabular}


other studies on the interference effect that have shown an underestimation of time. Contrast 2, comparing the two dual-task conditions, was not significant $(F<1)$, indicating that the two sequencing tasks were equally disruptive.

Absolute error. Timing responses were also transformed into mean percentage absolute error scores. Several studies suggest that this measure is sensitive in detecting timing error in dual-task situations (e.g., Brown \& Boltz, 2002; Brown \& West, 1990). The scores were formed by computing the absolute difference between each temporal production and the actual target interval $(5 \mathrm{sec})$ and dividing the result by the target interval; these values were then multiplied by 100 to represent the percentage by which temporal judgments deviated from accuracy. The average score was then computed for each subject in each task condition. A one-way repeated measures ANOVA performed on these scores uncovered a significant task effect $\left[F(2,116)=6.45, p<.002, \eta_{\mathrm{p}}^{2}=.10\right]$. The single-task $(M=28.0 \%)$ versus combined dual-task $(M=36.5 \%)$ comparison was significant $[F(1,58)=10.47, p<.002$, $\left.\eta_{\mathrm{p}}^{2}=.15\right]$; the two dual tasks, however, did not differ significantly $(F<1)$.

\section{Reasoning Performance}

Performance on the sequence-reasoning task was assessed in terms of speed and accuracy. Speed was given by the average RT associated with the various experimental conditions. Longer RTs would signify greater difficulty in processing the items. Accuracy on the task was determined by the percentage of items that were answered correctly. The mean RT and accuracy scores for the various experimental conditions are depicted in Table 3.

Response time. The mean RT in seconds for responding to the reasoning items was tabulated for each subject in each condition. These scores were submitted to a $2 \times 2$ repeated measures ANOVA. The factors were task (single task vs. dual task) and reasoning (positive vs. negative items). Both main effects were significant. The reasoning effect $\left[F(1,58)=113.83, p<.001, \eta_{\mathrm{p}}^{2}=.66\right]$ indicated that the negative items $(M=5.4 \mathrm{sec})$ were associated with longer RTs than were the positive items $(M=4.0 \mathrm{sec})$. This result is consistent with Baddeley's (1968) original findings and supports the idea that the negatively worded items are more difficult to process than the positively worded items. However, the main results concern the task effect $\left[F(1,58)=11.48, p<.001, \eta_{\mathrm{p}}^{2}=.16\right]$. The timing + reasoning dual task had longer RTs $(M=4.9 \mathrm{sec})$ than did the reasoning-only single task $(M=4.5 \mathrm{sec})$. This result shows that the concurrent timing task interfered with sequence-reasoning performance. The extra cognitive workload imposed by timing slowed down sequence processing, causing the subjects to spend more time with each item. The interaction was not significant $(F<1)$.

Percentage correct. Accuracy on the reasoning tasks was evaluated by analyzing percent correct scores. These scores correspond to the percentage of reasoning items that were answered correctly. Percent correct scores were calculated for each subject in each condition and were submitted to a $2 \times 2$ (task $\times$ reasoning) repeated measures ANOVA. In this case, the effect for task was not
Table 3

Mean Scores (and Standard Errors) for Two SequenceReasoning Performance Measures in Different Task and Reasoning Conditions in Experiment 1

\begin{tabular}{|c|c|c|c|c|}
\hline \multirow{3}{*}{$\begin{array}{c}\text { Task } \\
\text { Condition }\end{array}$} & \multicolumn{4}{|c|}{ Reasoning Condition } \\
\hline & \multicolumn{2}{|c|}{ Positive } & \multicolumn{2}{|c|}{ Negative } \\
\hline & $M$ & $S E$ & $M$ & $S E$ \\
\hline \multicolumn{5}{|c|}{ Response Time (sec) } \\
\hline Single task & 3.7 & 0.1 & 5.2 & 0.2 \\
\hline Dual task & 4.3 & 0.2 & 5.6 & 0.2 \\
\hline \multicolumn{5}{|c|}{ Percentage Correct } \\
\hline Single task & 77.7 & 2.1 & 74.6 & 2.1 \\
\hline Dual task & 80.6 & 2.2 & 75.1 & 2.3 \\
\hline
\end{tabular}

significant $(p>.10)$. The subjects were equally accurate under dual-task conditions as under single-task conditions. Overall, they averaged $77.3 \%$ correct. The analysis did, however, produce a significant effect for reasoning $\left[F(1,58)=19.35, p<.001, \eta_{\mathrm{p}}^{2}=.25\right]$, showing that the subjects were more accurate in responding to the positive items $(M=79.2 \%)$ than in responding to the negative items $(M=74.9 \%)$. This outcome is consistent with the result obtained with the $\mathrm{RT}$ scores. The task $\times$ reasoning interaction was not significant $(p>.20)$.

To summarize the results of Experiment 1, the data reveal a strong interference effect in timing. In comparison with timing-only conditions, the addition of a concurrent reasoning task caused temporal productions to become more variable, longer, and less accurate. As for reasoning performance, the subjects were significantly slower in processing the reasoning items under dual-task than under single-task conditions. This result indicates that concurrent timing interfered with sequence-reasoning performance. Although concurrent timing lengthened RTs, it did not produce a corresponding drop in accuracy. This pattern may be an example of the speed/accuracy tradeoff, an effect found in many motor, perceptual, and cognitive tasks (see, e.g., Meyer, Irwin, Osman, \& Kounois, 1988; Osman, et al., 2000). In this instance, the subjects sacrificed speed for accuracy. That is, the subjects spent more time processing each item in order to maintain the same level of accuracy under dual-task as under singletask conditions. It is important to note that a reduction in either speed or accuracy represents interference. Overall, the findings provide evidence of bidirectional interference, suggesting that timing and sequencing tasks rely on common resources.

\section{EXPERIMENT 2 Timing and Sequence Monitoring}

The sequencing task in Experiment 2 required the subjects to follow an ordered series of items displayed on the computer screen. The subjects were instructed to be on the lookout for any departures from the normal, expected sequence. This task was derived from two earlier versions of sequencing tasks. The first was the Trails Test, a subtest of the Halstead-Reitan Neuropsychological Battery (see Broshek \& Barth, 2000). There are two forms of the Trails Test. In Trails A, subjects use a pencil to join together (in 
order) a series of numbered squares that are scattered about on a sheet of paper. In Trails B, subjects join together an alternating sequence of numbers and letters (1-A, 2-B, $3-\mathrm{C}$, etc.). This test is sensitive at detecting frontal lobe damage, which typically involves problems with planning and sequencing behaviors. From this test, an alternation task was developed by Baddeley and his colleagues (Baddeley, 1996; Baddeley, Emslie, Kolodny, \& Duncan, 1998). Baddeley created a version of the task that would be more applicable for use in dual-task experiments. In this case, the subjects themselves produced an alternating series of letters and numbers by verbalizing the sequence. This task interfered with concurrent tasks that required keeping track of event order, such as a randomization task (Baddeley et al., 1998). Baddeley (1996) argued that the alternation task taps into the executive cognitive functions of scheduling and coordinating actions.

The present experiment involved our modification of the task. In this version, the item sequence was presented to the subjects, who then monitored that sequence to detect omissions in the series. There were at least two advantages of configuring the task as a monitoring task. The first was a matter of experimental control. We did not want the subjects to actually generate the item sequence, as in Baddeley's version, because we believed it would be too tempting to use it as a pacing strategy to help count out 5-sec intervals. That is, the subjects could adjust their verbal output to mark time. Instead, the experimenters controlled the item rate, not the subjects. The second advantage was that our task is essentially a signal detection task, with the omissions being the signals to detect. This situation allowed us to evaluate performance in terms of perceptual sensitivity and response bias, measures that otherwise would not be available.

\section{Method}

\section{Subjects}

Thirty-two students ( 9 male, 23 female) enrolled in general psychology classes volunteered for the experiment in exchange for extra credit. The average age of the students was 21.5 years $(S D=$ 4.2 years).

\section{Apparatus and Stimuli}

The computer hardware was the same as that used in Experiment 1 . The stimuli for the sequence-monitoring task consisted of a series of items displayed in the center of the computer screen at a rate of $1.2 \mathrm{sec} /$ item, which corresponded to 100 items for each 2 -min trial. Each succeeding item replaced the previous one, so that only 1 item was actually present on the screen at any given moment. These items were composed of letters or letter-number pairs (see below).

\section{Design and Procedure}

The experimental design was identical to that in the first experiment. The subjects were tested individually in two sessions separated by 1-2 weeks. Watches were removed prior to each session. Session 1 comprised the single-task conditions, in which the subjects performed a timing task, an alphabetic version of the sequencemonitoring task, and an alphanumeric version of the sequencemonitoring task. Session 2 involved the dual-task conditions, in which the subjects performed the timing and sequence-monitoring tasks concurrently. The subjects were told to assign equal priority to the two tasks. As in Experiment 1, each task condition was executed for three 2-min trials, and the order of the tasks within each session was counterbalanced across subjects.

The timing task was serial temporal production of 5-sec intervals. As before, the subjects were asked to generate these intervals via space bar presses continuously throughout each trial. There were two versions of the sequence-monitoring task. In the alphabetic $(\mathrm{AB})$ condition, the items presented on the screen were a series of letters in alphabetical order. The starting letter on any trial was selected randomly from the subset $\mathrm{A}$ through $\mathrm{O}$, inclusive. The subjects were instructed to press the Enter key on the keyboard whenever they detected a skip (an omission) in the alphabetic sequence. The subjects were encouraged to make these responses as quickly as possible. In the alphanumeric (AN) condition, the item sequence consisted of letter-number pairs in the form D-12, E-13, $\mathrm{F}-14$, and so forth. The starting number was selected randomly from 1 through 35, inclusive. In this case, the subjects were required to simultaneously monitor both the alphabetic series and the numeric series, because an omission could occur with either one. We reasoned that the AN task would probably be more demanding, because the subjects had to keep track of two separate sequence streams. The probability of an omission target was set at $p=.15$ (15 letters in each AB trial and 8 letters and 7 numbers in each AN trial). In both sequences, there was at least a two-item buffer separating omission targets.

\section{Results and Discussion}

\section{Timing Performance}

As in the first experiment, the timing data were converted into CV, mean IRI, and mean absolute error scores for analysis (see Table 4). As before, responses from the three trials were collapsed prior to deriving the performance measures. Analyses including trials as a factor did not produce differences appreciably different from those we report.

Coefficient of variation. CV scores were submitted to a one-way repeated measures ANOVA comparing the timing-only single-task, the timing $+\mathrm{AB}$ dual-task, and the timing + AN dual-task conditions. These conditions exerted a significant effect in the analysis $[F(2,62)=$ $\left.15.46, p<.001, \eta_{\mathrm{p}}^{2}=.33\right]$. Contrast $1[F(1,31)=28.54$, $\left.p<.001, \eta_{\mathrm{p}}^{2}=.48\right]$ confirmed that variability increased from the timing-only single-task condition $(M=0.15 \mathrm{sec})$

Table 4

Mean Scores (and Standard Errors) for Three Timing Performance Measures in Different Task Conditions in Experiment 2

\begin{tabular}{|c|c|c|c|c|c|c|}
\hline \multirow[b]{2}{*}{ Task Condition } & \multicolumn{2}{|c|}{$\begin{array}{l}\text { Coefficient of } \\
\text { Variation }(\mathrm{sec})\end{array}$} & \multicolumn{2}{|c|}{$\begin{array}{l}\text { Interresponse } \\
\text { Interval (sec) } \\
\end{array}$} & \multicolumn{2}{|c|}{$\begin{array}{l}\text { Percentage of } \\
\text { Absolute Error }\end{array}$} \\
\hline & $M$ & $S E$ & $M$ & $S E$ & $M$ & $S E$ \\
\hline Timing only & 0.15 & 0.01 & 4.6 & 0.3 & 26.4 & 2.6 \\
\hline Timing + alphabetic task & 0.20 & 0.02 & 5.3 & 0.2 & 26.4 & 2.4 \\
\hline Timing + alphanumeric task & 0.27 & 0.02 & 5.3 & 0.2 & 28.0 & 1.5 \\
\hline
\end{tabular}


to the combined dual-task conditions $(M=0.24 \mathrm{sec})$. Furthermore, Contrast $2\left[F(1,31)=7.78, p<.01, \eta_{\mathrm{p}}^{2}=.21\right]$ showed that the AN task $(M=0.27 \mathrm{sec})$ created more disruption than did the $\mathrm{AB}$ task $(M=0.20 \mathrm{sec})$. This pattern fits in with the idea that the more demanding the distractor task, the greater the diversion of attentional resources away from timing.

Interresponse interval. Mean IRI scores, signifying the average length of temporal productions, were computed for each subject and submitted to a one-way repeated measures ANOVA. The significant task effect $\left[F(2,62)=7.08, p<.002, \eta_{\mathrm{p}}^{2}=.19\right]$ was probed with orthogonal contrasts to test for differences between conditions. Contrast $1\left[F(1,31)=12.35, p<.001, \eta_{\mathrm{p}}^{2}=.28\right]$ revealed that the temporal productions lengthened from the timing-only condition $(M=4.6 \mathrm{sec})$ to the combined dual-task conditions $(M=5.3 \mathrm{sec})$. This pattern corresponds to the results of Experiment 1 and to the results of other studies on the interference effect that have shown an underestimation of time. Contrast 2 , comparing the two dual-task conditions, was not significant $(F<1)$.

Absolute error. Mean percentage absolute error scores were calculated for each subject and submitted to a oneway repeated measures ANOVA of the three task conditions. The task effect was not significant $(F<1)$. As can be seen in Table 4 , the three task conditions produced very similar levels of error in time judgments on this measure (combined $M=26.9 \%$ ).

\section{Detection Performance}

Error detection on the sequence-monitoring task was evaluated with three performance measures. The first measure was RT, the speed with which the subjects responded to omissions in the sequence. Two other measures, derived from signal detection theory, were used to assess perceptual sensitivity and response bias on the detection task. The mean scores for these measures are presented in Table 5.

Response time. The mean RT (in milliseconds) was calculated for each subject and cast in the form of a $2 \times 2$ repeated measures ANOVA. The factors were task (single task vs. dual task) and sequence (AB vs. AN). The primary finding concerns the effect for task $[F(1,31)=67.88, p<$ $\left..001, \eta_{\mathrm{p}}^{2}=.69\right]$, which indicated that the dual (timing + sequencing) tasks had longer RTs $(M=804 \mathrm{msec})$, relative to the single (sequencing-only) tasks $(M=739 \mathrm{msec})$. This result shows that the addition of a concurrent timing task interfered with sequencing performance, a result in line with the idea of resource sharing between the timing and the sequencing tasks. The sequence main effect $\left[F(1,31)=319.59, p<.001, \eta_{\mathrm{p}}^{2}=.91\right]$ showed that the AN sequence was associated with slower responding $(M=$ $857 \mathrm{msec})$, as compared with the $\mathrm{AB}$ sequence $(M=$ $686 \mathrm{msec})$. The significant task $\times$ sequence interaction $\left[F(1,31)=25.75, p<.001, \eta_{\mathrm{p}}^{2}=.45\right]$ was probed with tests of simple main effects. First, a comparison of the task conditions within each sequence showed that within the $\mathrm{AB}$ sequence, the dual task was associated with longer RTs, as compared with the single task $[F(1,31)=90.59$, $\left.p<.001, \eta_{\mathrm{p}}^{2}=.75\right]$. Within the AN sequence, the single versus dual comparison failed to reach significance $(p<$
Table 5

Mean Scores (and Standard Errors) for Three Sequence-

Monitoring Performance Measures in Different Task and Sequence Conditions in Experiment 2

\begin{tabular}{|c|c|c|c|c|}
\hline \multirow{3}{*}{$\begin{array}{c}\text { Task } \\
\text { Condition }\end{array}$} & \multicolumn{4}{|c|}{ Sequence Condition } \\
\hline & \multicolumn{2}{|c|}{ Alphabetic } & \multicolumn{2}{|c|}{ Alphanumeric } \\
\hline & $M$ & $S E$ & $M$ & $S E$ \\
\hline \multicolumn{5}{|c|}{ Response Time (msec) } \\
\hline Single task & 633 & 14 & 846 & 12 \\
\hline Dual task & 738 & 14 & 869 & 13 \\
\hline \multicolumn{5}{|c|}{ Perceptual Sensitivity $\left(A^{\prime}\right)$} \\
\hline Single task & .983 & .005 & .863 & .008 \\
\hline Dual task & .895 & .010 & .808 & .012 \\
\hline \multicolumn{5}{|c|}{ Response Bias $\left(B_{\mathrm{D}}^{\prime \prime}\right)$} \\
\hline Single task & .207 & .148 & .943 & .007 \\
\hline Dual task & .868 & .019 & .948 & .030 \\
\hline
\end{tabular}

.06). Second, comparisons of the sequence conditions within each task confirmed that AN sequence produced longer RTs for both the single $[F(1,31)=367.24, p<$ $\left.\left..001, \eta_{\mathrm{p}}^{2}=.92\right)\right]$ and the dual $[F(1,31)=88.10, p<.001$, $\left.\eta_{\mathrm{p}}^{2}=.74\right]$ tasks.

Perceptual sensitivity. As was noted previously, the sequence-monitoring task represents a signal detection situation, in which subjects are on the lookout for omission errors that occur against a noise background. In this context, a hit is defined as a response occurring when an out-of-sequence item is displayed on the screen; a false alarm is a response to an in-sequence item. These responses were used to create independent measures of sensitivity and bias. One common measure of perceptual sensitivity is $A^{\prime}$ (Grier, 1971), a nonparametric statistic that ranges in value from 1.000 (maximum sensitivity) to 0.500 (maximum insensitivity). $A^{\prime}$ scores were calculated for each subject and submitted to a $2 \times 2$ (task $\times$ sequence) repeated measures ANOVA. The main effect for task $\left[F(1,31)=118.28, p<.001, \eta_{\mathrm{p}}^{2}=.80\right]$ showed that the dual-task condition $(M=.852)$ had lower sensitivity scores than did the single-task condition $(M=.923)$. This result provides strong evidence that the timing task interfered with the sequencing task. The sequence main effect $\left[F(1,31)=183.66, p<.001, \eta_{\mathrm{p}}^{2}=.85\right]$ showed that sensitivity declined from the AB sequence $(M=.939)$ to the AN sequence $(M=.836)$. This result makes sense, assuming that the AN sequence is more difficult to track. The task $\times$ sequence interaction $[F(1,31)=4.23, p<.05$, $\left.\eta_{\mathrm{p}}^{2}=.13\right]$ served to reinforce these findings. Simple main effects tests confirmed that, as compared with the singletask condition, sensitivity was lower under the dual-task conditions for both the $\mathrm{AB}[F(1,31)=124.34, p<.001$, $\left.\eta_{\mathrm{p}}^{2}=.80\right]$ and the AN $\left[F(1,31)=19.00, p<.001, \eta_{\mathrm{p}}^{2}=\right.$ .39] sequences. Likewise, sensitivity dropped from the AB to the $\mathrm{AN}$ sequence under both the single-task $[F(1,31)=$ $\left.276.25, p<.001, \eta_{\mathrm{p}}^{2}=.89\right]$ and the dual-task $[F(1,31)=$ $\left.38.20, p<.001, \eta_{\mathrm{p}}^{2}=.55\right]$ conditions.

Response bias. Response bias refers to a general tendency to report "yes, I detect a target" (liberal bias) or "no, I do not detect a target" (conservative bias) when one is uncertain. Response bias was measured with a 
nonparametric statistic called $B_{\mathrm{D}}^{\prime \prime}$ (Donaldson, 1992). $B_{\mathrm{D}}^{\prime \prime}$ ranges in value from -1.000 (maximum liberal bias) to 0 (no bias) to +1.000 (maximum conservative bias). These scores were computed for each subject and submitted to a task $\times$ sequence repeated measures ANOVA. The results conformed to some basic findings in the signal detection literature. First, all of the mean scores were positive values, indicating that the subjects tended to be conservative in their judgments (i.e., responding only when they were certain that an omission had occurred). This outcome is typical of detection experiments in which the probability of a signal is low (in the present case, $p=.15$ ). Another standard finding is that subjects tend to become more conservative (i.e., to adopt a strict criterion) when task demands increase (Brown, 1997; Brown \& Boltz, 2002; Schneider \& Fisk, 1982). That process was reflected in the task main effect $\left[F(1,31)=19.68, p<.001, \eta_{\mathrm{p}}^{2}=\right.$ $.39]$, which showed that the dual task $(M=.908)$ was associated with a more conservative bias (i.e., higher $B_{\mathrm{D}}^{\prime \prime}$ scores), as compared with the single task $(M=.575)$. The sequence main effect $\left[F(1,31)=25.57, p<.001, \eta_{\mathrm{p}}^{2}=\right.$ $.45]$ was compounded by the task $\times$ sequence interaction $\left[F(1,31)=20.44, p<.001, \eta_{\mathrm{p}}^{2}=.40\right]$. Simple main effects tests indicated that the $\mathrm{AB}$ and the $\mathrm{AN}$ sequences differed only in the single-task condition $[F(1,26)=13.78$, $p<.001]$. As is shown in Table 5, $B_{\mathrm{D}}^{\prime \prime}$ scores in the dualtask condition remained uniformly near the ceiling of conservative bias for both sequences $(F<1)$. Comparisons of the two sequences within each task condition showed that scores were higher for the $\mathrm{AN}$ than for the $\mathrm{AB}$ sequence in both the single-task condition $[F(1,31)=24.36, p<$ $\left..001, \eta_{\mathrm{p}}^{2}=.44\right]$ and the dual-task condition $[F(1,31)=$ 4.91, $\left.p<.04, \eta_{\mathrm{p}}^{2}=.13\right]$. This pattern is consistent with the idea that the AN sequence is more demanding.

In sum, Experiment 2 showed interference in timing in the form of greater variability and a lengthening in time judgments under dual-task conditions. There was also compelling evidence that the concurrent timing task interfered with sequence detection. In comparison with single-task conditions, under dual-task conditions, the subjects tended to be slower, less sensitive, and more conservative at detection.

\section{GENERAL DISCUSSION}

This research provides information on the issue of the attentional resources involved in temporal information processing. The two experiments yielded similar patterns of results, despite the fact that seemingly, the distractor tasks involved were different. These differences (e.g., verbal reasoning versus target detection) were only superficial, however, since both tasks were fundamentally centered on the perception and judgment of event sequences. The results point to the role of sequence processing as an important element in timing and suggest the operation of common underlying mechanisms.

One basic finding concerns dual-task interference in timing. In both experiments, the concurrent sequencing tasks disrupted timing performance. In each case, temporal productions were more variable (as reflected in $\mathrm{CV}$ scores) under dual-task (timing + sequencing) conditions than under single-task (timing-only) conditions. Temporal productions also became longer under dual-task conditions in both experiments, indicating a shift toward a shortening of perceived time. These results demonstrate the classic interference effect in timing. The absolute error measure produced a mixed result, detecting a dual-task decrement in one experiment (Experiment 1), but not in the other (Experiment 2).

The role of timekeeping strategies, such as chronometric counting in the interference effect, deserves comment. It is likely that under the timing-only conditions, the subjects spontaneously used counting and/or some other method, since "these strategies seem very much a part of the natural mechanism" (Poynter, 1989, p. 310) for tracking time. Counting is an effective strategy for judging time, since it improves accuracy and reduces variability (e.g., Grondin, Meilleur-Wells, \& Lachance, 1999; Grondin, Ouellet, \& Roussel, 2004). Counting involves explicitly dividing the interval into a series of 1-sec temporal productions, and as with any other timing task, it uses attentional resources. A distractor task draws attention away from these strategies. However, the interference effect is not simply due to the prevention of counting. Subjects still show interference when judging intervals that are too short to be timed with a counting strategy (e.g., Casini \& Ivry, 1999; Chastain \& Ferraro, 1997; Thomas \& Cantor, 1978). Timing, whether accompanied by chronometric counting or not, is a resourcedemanding task, and anything that diverts attention away will probably produce interference.

The timing data showed two additional features that warrant some discussion. The first feature concerns the accuracy of the temporal productions. The subjects in the dual-task conditions in Experiment 1 generated temporal productions that were actually very close to the 5 -sec target interval $(M=5.1 \mathrm{sec})$, whereas productions in the singletask control condition were less accurate $(M=4.4 \mathrm{sec})$. This result is not unusual. The single-task situation allows subjects to focus on time without distraction, a condition referred to as "the experience of time-in-passing" (Hicks, Miller, Gaes, \& Bierman, 1977, p. 431); it is well established that such conditions evoke a substantial lengthening of perceived time. The short temporal productions we obtained correspond to this expected lengthening effect. The dual-task conditions shorten perceived time, leading to the longer temporal productions we report. As with many timing studies, the critical issue here is a relative comparison of time judgments under different experimental conditions, rather than an evaluation of accuracy per se (Ornstein, 1969). In Experiment 2, the average production (IRI) scores ( $M$ single task $=4.6 \mathrm{sec}$ and $M$ dual task $=$ $5.3 \mathrm{sec}$ ) showed the same relative difference, although both scores in this case deviated from accuracy. In each experiment, however, the dual tasks showed the same relative $15 \%-16 \%$ increase in the lengthening of perceived time, as compared with the single tasks.

The second feature to note is that manipulations of the difficulty of the sequencing tasks did not exert much influence on timing performance. In most instances, the easier and more difficult versions of the tasks disrupted concur- 
rent timing to a similar degree. This lack of an effect for task difficulty corresponds to what resource theorists term difficulty insensitivity (see Wickens, 1980, 1984). One explanation for difficulty insensitivity is that the resources affected by increases in the difficulty level of Task A play only a minimal (or nonexistent) role in the performance of concurrent Task B. In the present research, attentional resources related to temporal information processing represent the critical resources relevant for both the sequencing and the timing tasks. In Experiment 1, although the negatively worded items were more difficult to process than the positively worded items (as indicated by RT and accuracy measures), this increase in difficulty may have been due to a greater involvement of semantic-processing resources, whereas the resource demand for temporal information processing remained essentially constant. A similar account may be applied to the alphabetic versus alphanumeric conditions in Experiment 2, although, in this case, the alphanumeric task requirements (monitoring two simultaneous sequences) may have led to some increased demand for temporal-processing resources, as reflected in increased variability in the temporal productions.

The fundamental issue of bidirectional interference hinges on performance on the sequencing tasks under single- and dual-task conditions. Most measures showed that the timing task interfered with sequencing. That is, as compared with sequencing-only conditions, the addition of a concurrent timing task disrupted performance. In Experiment 1, the concurrent timing task substantially slowed sequence-reasoning responses. In this case, the dual-task condition produced a speed/accuracy tradeoff. The subjects were able to maintain accuracy on the sequence-reasoning task from single-task to dual-task conditions, but the preservation of accuracy came at a cost of reduced reasoning speed. Thus, fewer problems were completed under the dual-task conditions. The sequence detection task in Experiment 2 provided a clear pattern. The concurrent timing task lengthened RTs to omission targets, reduced perceptual sensitivity at detecting the targets, and prompted the subjects to become more conservative in their judgments. Overall, the results offer good evidence for bidirectional interference. The findings suggest that the timing and sequencing tasks invoke related information-processing mechanisms and, so, draw from the same pool of attentional resources.

Can the interference results be explained in terms of response conflict, rather than resource allocation? Given that the timing and sequencing tasks in both experiments involved motor responses, bidirectional interference may have arisen from conflicts within the motor system. For example, longer RTs in the dual-task conditions may have simply been an artifact, if one response was delayed until the other was completed. Several points argue against this interpretation. First, the motor responses involved were minimal. The responses were limited to simple buttonpresses executed with different hands and were unlikely to have overloaded manual resources. As was described previously, aviation researchers have used temporal production as a secondary task because it tends not to interfere with piloting performance. In the dual-task literature, most instances of motor response conflict involve more complex movement tasks, such as manual tracking, precision aiming, perceptual-motor incompatibility, and so forth (e.g., Damos, Bittner, Kennedy, \& Harbeson, 1981; Fischer, 1997; Tsang, Velazquez, \& Vidulich, 1996; Wickens, 1980). Second, even if the subjects were unable to execute simultaneous responses, the timing task was unlikely to have been affected significantly by a concurrent sequencing task, because of the magnitude of the durations involved. The subjects attempted to produce 5 -sec target intervals, and any response delays caused by closein-time sequencing responses would have been very small, relative to the length of the temporal production. Furthermore, the interference effect has been demonstrated with a wide variety of timing and distractor tasks in different experimental paradigms, which precludes an explanation based on motor interference (Brown, 1997). Third, the magnitude of the sequencing responses in Experiment 1 makes it unlikely that they were influenced substantially by the timing responses. As with the timing responses, the sequencing response times were on the order of 4-5 sec $(M$ single task $=4.5 \mathrm{sec}, M$ dual task $=4.9 \mathrm{sec})$; thus, any delays due to simultaneous timing responses would have been minimal. The RTs for the sequencing task in Experiment 2 were much shorter $(M$ single $=739 \mathrm{msec}$, $M$ dual $=804 \mathrm{msec}$ ). Given that the mean temporal production in the dual-task condition was $5.3 \mathrm{sec}$, the subjects made an average of over 6.6 sequencing responses for every timing response, which severely limited the number of instances of simultaneous motor responses. Despite the large difference in the magnitude of the RTs for the sequencing tasks in the two experiments, there was a remarkably similar proportional increase in RTs from single- to dual-task conditions, amounting to an $8.2 \%$ increase in Experiment 1 and 8.1\% in Experiment 2. Finally, a motor conflict explanation cannot account for the effects on sensitivity and bias obtained in Experiment 2. All these factors suggest that the interference effects we report were essentially cognitive in nature, rather than motor.

The main findings suggest that timing and sequencing involve the same (or similar) cognitive processes. Not only are timing and sequencing conceptually related, but they are theoretically associated as well. Cognitive theorists have developed various models of executive processing in which temporal functions, such as the sequencing of responses and coordination of actions, play a major role. One influential model is the supervisory attentional system (SAS), which describes an attentional controller mechanism that integrates multiple inputs, coordinates dual-task performance, and schedules responses (Norman \& Shallice, 1986; Shallice \& Burgess, 1996). Many of the features of the SAS have been incorporated into the working memory (WM) model (Baddeley, 1992a, 1992b, 1994; Baddeley \& Hitch, 1974), a multicomponent, multiple resource model of short-term memory. Baddeley believes that scheduling is handled by the central executive part of WM. The central executive serves as an attentional supervisor mechanism. It is the component that monitors and directs ongoing behavior, integrates and coordinates information, and is responsible for planning and organiz- 
ing actions (Baddeley, 1992a, 1992b). Baddeley (1990, pp. 132-135; Baddeley et al., 1998) argued that tracking an ordered response sequence places heavy demands on executive resources. These theoretical models offer a framework for investigating sequencing and timing processes. In line with this view are dual-task experiments that show bidirectional interference between time judgments and a variety of other executive-level tasks, particularly those related to sequence processing, such as memory updating (Brown \& Frieh, 2000), digit order memory (Shinohara, 1999), and random number generation (Brown, 2006). These findings point to an underlying commonality between judgments of duration, order, and sequence.

\section{AUTHOR NOTE}

Portions of these data were presented at the meetings of the New England Sequencing and Timing (NEST) conference, Yale University, New Haven, CT, in March 2003 and March 2004. The authors thank Simon Grondin, Françoise Macar, and two anonymous reviewers for their comments on an earlier draft of the manuscript. Correspondence should be addressed to S. W. Brown, Department of Psychology, University of Southern Maine, Portland, ME 04104-9300 (e-mail: swbrown@usm.maine.edu).

\section{REFERENCES}

BADDELEy, A. D. (1968). A 3 min reasoning test based on grammatical transformation. Psychonomic Science, 10, 341-342.

BADDELEY, A. [D.] (1990). Human memory: Theory and practice. Boston: Allyn \& Bacon.

BADDELEY, A. D. (1992a). Is working memory working? The fifteenth Bartlett lecture. Quarterly Journal of Experimental Psychology, 44A, $1-31$.

BADDELEy, A. D. (1992b). Working memory. Science, 255, 556-559.

BADDEleY, A. D. (1994). Working memory: The interface between memory and cognition. In D. L. Schacter \& E. Tulving (Eds.), Memory systems 1994 (pp. 351-367). Cambridge, MA: MIT Press.

BADDELEY, A. [D.] (1996). Exploring the central executive. Quarterly Journal of Experimental Psychology, 49A, 5-28.

Baddeley, A. [D.], Emslie, H., Kolodny, J., \& Duncan, J. (1998). Random generation and the executive control of working memory. Quarterly Journal of Experimental Psychology, 51A, 819-852.

BADDELEY, A. D., \& Hitch, G. (1974). Working memory. In G. H. Bower (Ed.), The psychology of learning and motivation (Vol. 8, pp. 47-89). New York: Academic Press.

BINDRA, D., \& WAKSBERG, H. (1956). Methods and terminology in studies of time estimation. Psychological Bulletin, 53, 155-159.

BLOCK, R. A. (1982). Temporal judgments and contextual change. Journal of Experimental Psychology: Learning, Memory, \& Cognition, 8, 530-544.

BLock, R. A. (1990). Models of psychological time. In R. A. Block (Ed.), Cognitive models of psychological time (pp. 1-35). Hillsdale, NJ: Erlbaum

BLOCK, R. A., \& ZAKAY, D. (1996). Models of psychological time revisited. In H. Helfrich (Ed.), Time and mind (pp. 171-195). Seattle, WA: Hogrefe \& Huber.

Bortolussi, M. R., Kantowitz, B. H., \& Hart, S. G. (1986). Measuring pilot workload in a motion base trainer: A comparison of four techniques. Applied Ergonomics, 17, 278-283.

BrosheK, D. K., \& Barth, J. (2000). The Halstead-Reitan Neuropsychological Test Battery. In G. Groth-Marnat (Ed.), Neuropsychological assessment in clinical practice: A guide to test interpretation and integration (pp. 223-262). New York: Wiley.

Brown, S. W. (1995). Time, change, and motion: The effects of stimulus movement on temporal perception. Perception \& Psychophysics, 57, 105-116.

BRown, S. W. (1997). Attentional resources in timing: Interference effects in concurrent temporal and nontemporal working memory tasks. Perception \& Psychophysics, 59, 1118-1140.
Brown, S. W. (1998). Automaticity versus timesharing in timing and tracking dual-task performance. Psychological Research, 61, 71-81.

Brown, S. W. (2006). Timing and executive function: Bidirectional interference between concurrent temporal production and randomization tasks. Memory \& Cognition, 34, 1464-1471.

Brown, S. W., \& BenNETT, E. D. (2002). The role of practice and automaticity in temporal and nontemporal dual-task performance. Psychological Research, 66, 80-89.

Brown, S. W., \& Boltz, M. G. (2002). Attentional processes in time perception: Effects of mental workload and event structure. Journal of Experimental Psychology: Human Perception \& Performance, 28, 600-615.

BRown, S. W., \& FrIEH, C. T. (2000). Information processing in the central executive: Effects of concurrent temporal production and memory updating tasks. In P. Desain \& L. Windsor (Eds.), Rhythm perception and production (pp. 193-196). Lisse, The Netherlands: Swets \& Zeitlinger.

Brown, S. W., Newcomb, D. C., \& KahrL, K. G. (1995). Temporal-signal detection and individual differences in timing. Perception, 24, 525-538.

Brown, S. W., \& WeSt, A. N. (1990). Multiple timing and the allocation of attention. Acta Psychologica, 75, 103-121.

Casali, J. G., \& Wierwille, W. W. (1983). A comparison of rating scale, secondary-task, physiological, and primary-task workload estimation techniques in a simulated flight task emphasizing communications load. Human Factors, 25, 623-641.

CAsali, J. G., \& Wierwille, W. W. (1984). On the measurement of pilot perceptual workload: A comparison of assessment techniques addressing sensitivity and intrusion issues. Ergonomics, 27, 1033-1050.

Casini, L., \& IVRY, R. B. (1999). Effects of divided attention on temporal processing in patients with lesions of the cerebellum or frontal lobe. Neuropsychology, 13, 10-21.

CASINI, L., \& MACAR, F. (1997). Effects of attention manipulation on judgments of duration and of intensity in the visual modality. Memory \& Cognition, 25, 812-818.

Chastain, G., \& Ferraro, F. R. (1997). Duration ratings as an index of processing resources required for cognitive tasks. Journal of General Psychology, 124, 49-76.

Damos, D. L., Bittner, A. C., Kennedy, R. S., \& Harbeson, M. M. (1981). Effects of extended practice on dual-task tracking performance. Human Factors, 23, 627-631.

DONALDSON, W. (1992). Measuring recognition memory. Journal of Experimental Psychology: General, 121, 275-277.

Doob, L. W. (1971). Patterning of time. New Haven, CT: Yale University Press.

Eggemeier, F. T., \& Wilson, G. F. (1991). Performance-based and subjective assessment of workload in multi-task environments. In D. L. Damos (Ed.), Multiple-task performance (pp. 217-278). London: Taylor \& Francis.

FinK, A., \& Neubauer, A. C. (2001). Speed of information processing, psychometric intelligence, and time estimation as an index of cognitive load. Personality \& Individual Differences, 30, 1009-1021.

Fischer, M. H. (1997). Attention allocation during manual movement preparation and execution. European Journal of Cognitive Psychology, 9, 17-51.

ForTIN, C. (1999). Short-term memory in time interval production. International Journal of Psychology, 34, 308-316.

Fortin, C., Duchet, M.-L., \& Rousseau, R. (1996). Tapping sensitivity to processing in short-term memory. Canadian Journal of Experimental Psychology, 50, 402-407.

Fortin, C., \& MASSE, N. (2000). Expecting a break in time estimation: Attentional time-sharing without concurrent processing. Journal of Experimental Psychology: Human Perception \& Performance, 26, 1788-1796.

Fortin, C., \& Rousseau, R. (1987). Time estimation as an index of processing demand in memory search. Perception \& Psychophysics, 42, 377-382.

Fournier, S., Larigauderie, P., \& GaOnaC'H, D. (2004). Exploring how the central executive works: A search for independent components. Psychologica Belgica, 44, 159-188.

Fraisse, P. (1963). The psychology of time (J. Leith, Trans.). New York: Harper \& Row.

Fraisse, P. (1978). Time and rhythm perception. In E. C. Carterette \& M. P. Friedman (Eds.), Handbook of perception: Vol. VIII. Perceptual coding (pp. 203-254). New York: Academic Press. 
Franssen, V., \& Vandierendonck, A. (2002). Time estimation: Does the reference memory mediate the effect of knowledge of results? Acta Psychologica, 109, 239-267.

Gibson, J. J. (1975). Events are perceivable but time is not. In J. T. Frasier \& N. Lawrence (Eds.), The study of time II (pp. 295-301). New York: Springer.

Goldstone, S., Boardman, W. K., \& Lhamon, W. T. (1958). Kinesthetic cues in the development of time concepts. Journal of Genetic Psychology, 93, 185-190.

GrIER, J. B. (1971). Nonparametric indexes for sensitivity and bias: Computing formulas. Psychological Bulletin, 75, 424-429.

Grondin, S., \& MaCar, F. (1992). Dividing attention between temporal and nontemporal tasks: A performance operating characteristicPOC - analysis. In F. Macar, V. Pouthas, \& W. J. Friedman (Eds.), Time, action, and cognition: Towards bridging the gap (pp. 119-128). Dordrecht: Kluwer.

Grondin, S., Meilleur-Wells, G., \& Lachance, R. (1999). When to start explicit counting in a time-intervals discrimination task: A critical point in the timing process of humans. Journal of Experimental Psychology: Human Perception \& Performance, 25, 993-1004.

Grondin, S., Ouellet, B., \& Roussel, M.-E. (2004). Benefits and limits of explicit counting for discriminating temporal intervals. $\mathrm{Ca}$ nadian Journal of Experimental Psychology, 58, 1-12.

GUAY, M., \& WILBERG, R. B. (1983). Immediate reproduction of temporal information under four cognitive strategies. Perceptual \& Motor Skills, 56, 375-381.

Hicks, R. E., Miller, G. W., Gaes, G., \& Bierman, K. (1977). Concurrent processing demands and the experience of time-in-passing. American Journal of Psychology, 90, 431-446.

Hiscock, M., Cheesman, J., Inch, R., Chipuer, H. M., \& Graff, L. A. (1989). Rate and variability of finger tapping as measures of lateralized concurrent task effects. Brain \& Cognition, 10, 87-104

Kantowitz, B. H., \& KNIGHT, J. L. (1974). Testing tapping timesharing. Journal of Experimental Psychology, 103, 331-336.

Kee, D. W., Morris, K., Bathurst, K., \& Hellige, J. B. (1986). Lateralized interference in finger tapping: Comparisons of rate and variability measures under speed and consistency tapping instructions. Brain \& Cognition, 5, 268-279.

Logan, G. D. (1985). Executive control of thought and action. Acta Psychologica, 60, 193-210.

MACAR, F. (2002). Expectancy, controlled attention and automatic attention in prospective temporal judgments. Acta Psychologica, 111, 243-262.

Macar, F., Grondin, S., \& CASINI, L. (1994). Controlled attention sharing influences time estimation. Memory \& Cognition, 22, 673-686.

Meyer, D. E., Irwin, D. E., Osman, A. M., \& Kounois, J. (1988). The dynamics of cognition and action: Mental processes inferred from speed-accuracy decomposition. Psychological Review, 95, 183-237.

Miyake, A., Friedman, N. P., Emerson, M. J., Witzki, A. H., Howerter, A., \& Wager, T. D. (2000). The unity and diversity of executive functions and their contributions to complex "frontal lobe" tasks: A latent variable analysis. Cognitive Psychology, 41, 49-100.

Navon, D., \& Gopher, D. (1979). On the economy of the humanprocessing system. Psychological Review, 86, 214-253.

NAVON, D., \& Gopher, D. (1980). Task difficulty, resources, and dualtask performance. In R. S. Nickerson (Ed.), Attention and performance VIII (pp. 297-315). Hillsdale, NJ: Erlbaum.

Norman, D. A., \& Shallice, T. (1986). Attention to action: Willed and automatic control of behavior. In R. J. Davidson, G. E. Schwartz, \& D. Shapiro (Eds.), Consciousness and self-regulation (Vol. 4,pp. 1-18). New York: Plenum.

O’Donnell, R. D., \& EgGemeier, F. T. (1986). Workload assessment methodology. In K. R. Boff, L. Kaufman, \& J. P. Thomas (Eds.), Handbook of perception and human performance: Vol. II. Cognitive processes and performance (pp. 42.1-42.49). New York: Wiley.

Ornstein, R. (1969). On the experience of time. New York: Penguin.

Osman, A., Lou, L., Müller-Gethmann, H., Rinkenauer, G., Mattes, S., \& UlRich, R. (2000). Mechanisms of speed-accuracy tradeoff: Evidence from covert motor processes. Biological Psychology, 51, 173-199.

PoYNTER, W. D. (1989). Judging the duration of time intervals: A pro- cess of remembering segments of experience. In I. Levin \& D. Zakay (Eds.), Time and human cognition: A life-span perspective (pp. 305321). Amsterdam: North-Holland.

Poynter, W. D., \& Homa, D. (1983). Duration judgment and the experience of change. Perception \& Psychophysics, 33, 548-560.

Royall, D. R., \& Mahurin, R. K. (1996). Neuroanatomy, measurement, and clinical significance of the executive cognitive functions. American Psychiatric Press Review of Psychiatry, 15, 175-204.

SaWyer, T. F., Meyers, G. J., \& Huser, S. J. (1994). Contrasting task demands alter the perceived duration of brief time intervals. Perception \& Psychophysics, 56, 649-657.

SCHNEIDER, W., \& Fisk, A. D. (1982). Concurrent automatic and controlled visual search: Can processing occur without resource cost? Journal of Experimental Psychology: Learning, Memory, \& Cognition, 8, 261-278.

Shallice, T., \& Burgess, P. (1996). The domain of supervisory processes and temporal organization of behaviour. Philosophical Transactions of the Royal Society of London: Series B, 351, 14051412.

ShinohaRa, K. (1999). Resource for temporal information processing in interval production. Perceptual \& Motor Skills, 88, 917-928.

Shinohara, K., Miura, T., \& Usui, S. (2002). Tapping task as an index of mental workload in a time sharing task. Japanese Psychological Research, 44, 144-151.

Thomas, E. A. C., \& Brown, I., JR. (1974). Time perception and the filled-duration illusion. Perception \& Psychophysics, 16, 449-458

Thomas, E. A. C., \& Cantor, N. E. (1978). Interdependence between the processing of temporal and non-temporal information. In J. Requin (Ed.), Attention and performance VII (pp. 43-62). Hillsdale, NJ: Erlbaum.

Tsang, P. S., Shaner, T. L., \& Vidulich, M. A. (1995). Resource scarcity and outcome conflict in time-sharing performance. Perception \& Psychophysics, 57, 365-378.

Tsang, P. S., Velazquez, V. L., \& Vidulich, M. A. (1996). Viability of resource theories in explaining time-sharing performance. Acta Psychologica, 91, 175-206.

WiCKENS, C. D. (1980). The structure of attentional resources. In R. S. Nickerson (Ed.), Attention and performance VIII (pp. 239-257). Hillsdale, NJ: Erlbaum.

Wickens, C. D. (1984). Processing resources in attention. In R. Parasuraman \& D. R. Davies (Eds.), Varieties of attention (pp. 63-102). New York: Academic Press.

WierWILle, W. W., \& CONNOR, S. A. (1983). Evaluation of 20 workload measures using a psychomotor task in a moving-base aircraft simulator. Human Factors, 25, 1-16.

Wierwille, W. W., Rahimi, M., \& Casali, J. G. (1985). Evaluation of 16 measures of mental workload using a simulated flight task emphasizing mediational activity. Human Factors, 27, 489-502.

ZAKAY, D. (1989). Subjective time and attentional resource allocation: An integrated model of time estimation. In I. Levin \& D. Zakay (Eds.), Time and human cognition: A life-span perspective (pp. 365-397). Amsterdam: North-Holland.

ZAKAY, D. (1993). Time estimation methods-Do they influence prospective duration estimates? Perception, 22, 91-101.

ZAKAY, D. (1998). Attention allocation policy influences prospective timing. Psychonomic Bulletin \& Review, 5, 114-118.

ZAKAY, D., \& Block, R. A. (1996). The role of attention in time estimation processes. In M. A. Pastor \& J. Artieda (Eds.), Time, internal clocks, and movement (pp. 143-164). Amsterdam: Elsevier.

ZAKAY, D., \& BLOCK, R. A. (1997). Temporal cognition. Current Directions in Psychological Science, 6, 12-16.

Zakay, D., Nitzan, D., \& Glicksohn, J. (1983). The influence of task difficulty and external tempo on subjective time estimation. Perception \& Psychophysics, 34, 451-456.

ZAKAY, D., \& Shub, J. (1998). Concurrent duration production as a workload measure. Ergonomics, 41, 1115-1128.

(Manuscript received March 23, 2006; revision accepted for publication August 16, 2006.) 